\title{
Widely used mutants of eiger, encoding the Drosophila Tumor Necrosis factor, carry additional mutations in the NimrodC1 phagocytosis receptor
}

\author{
Albana Kodra $^{1 *}$, Claire de la Cova ${ }^{2 \star}$, Abigail R. Gerhold ${ }^{3 *}$ and Laura A. Johnston ${ }^{1 \rrbracket}$ \\ ${ }^{1}$ Department of Genetics \& Development, Columbia University Irving Medical Center, \\ Vagelos College of Physicians and Surgeons, New York, NY \\ 2 Department of Biological Sciences, University of Wisconsin, Milwaukee, WI \\ ${ }^{3}$ Department of Biology, McGill University, Montréal, QC \\ * These authors made equal contributions \\ I Address correspondence to Laura A. Johnston, lj180@columbia.edu
}

\begin{abstract}
The process of apoptosis in epithelia involves activation of caspases, delamination of cells, and degradation of cellular components. Corpses and cellular debris are then rapidly cleared from the tissue by phagocytic blood cells. In studies of the Drosophila TNF, Eiger (Egr) and cell death in wing imaginal discs, the epithelial primordia of fly wings, we noticed that dying cells persisted longer in egr ${ }^{3}$ mutant wing discs than in wild type discs, raising the possibility that their phagocytic engulfment by hemocytes was impaired. Further investigation revealed that lymph glands and circulating hemocytes from egr ${ }^{3}$ mutant larvae were completely devoid of NimC1 staining, a marker of phagocytic hemocytes. Genome sequencing uncovered mutations in the NimC1 coding region that are predicted to truncate the NimC1 protein before its transmembrane domain, and provide an explanation for the lack of NimC staining. The work that we report here demonstrates the presence of these NimC1 mutations in the widely used egr $r^{3}$ mutant, its sister allele, egr $r^{1}$, and its parental strain, Regg $1^{G S 9830}$. As the egr ${ }^{3}$ and egr ${ }^{1}$ alleles have been used in numerous studies of immunity and cell death, it may be advisable to re-evaluate their associated phenotypes.
\end{abstract}

\section{Results and Discussion}

The Drosophila genome encodes a single TNF homolog, known as Eiger (Eda-like cell death trigger, Egr) (IGAKI et al. ; MORENO et al. ; NARASIMAMURTHY et al.). Egr is expressed in many different tissues and plays various roles in cellular processes such as 
the immune response, energy homeostasis, and JNK-dependent cell death. Since its identification, numerous studies on cell death and immunity have utilized the egr ${ }^{3}$ and egr ${ }^{1}$ alleles, which were generated by imprecise excisions of the Regg $1^{\mathrm{GS} 9830 \mathrm{P} \text { element }}$ and resulted in deletions of the first coding exon of the egr gene (IGAKI et al.). Both egr ${ }^{3}$ and egr ${ }^{1}$ strains are homozygous viable and considered severe loss-of-function alleles.

Dead cells in Drosophila are commonly removed from tissues by phagocytic engulfment by plasmatocytes, the most abundant of the circulating hemocytes in the larva (ABRAMS et al. ; SONNENFELD AND JACOBS ; FRANC et al. ; SHKLYAR et al.). Plasmatocytes carry cell surface receptors for the recognition and rapid engulfment of bacteria, dead cells and cellular debris, such as Eater (KOCKS et al. ; CHUNG AND KoCKS), NimrodC1 (NimC1) (KURUCZ et al. ; HONTI et al.) and Draper (MANAKA et al.). A frequently used marker for plasmatocytes in Drosophila is positivity for NimC1, a transmembrane protein characterized by the presence of a special type of EGF repeat known as the NIM repeat, located immediately proximal to a conserved CCXGY motif (SOMOGYl et al.). The NimC1 gene is part of a cluster of four $\mathrm{NimC}$ (NimC1-4) genes in the midst of several other related Nimrod genes at 34E on chromosome 2. Nimrod proteins contain 2-16 NIM repeats as well as additional conserved residues at their amino terminus. The Nimrod proteins, together with Eater and Draper, form a conserved superfamily of 12 proteins in Drosophila, and Nimrod proteins are also encoded in the $C$. elegans and mammalian genomes (MELCARNE et al.). Loss of any Nimrod protein diminishes the capacity of hemocytes to fight microbes. For example, RNAi against NimC1 has implicated it in bacterial phagocytosis (KURUCZ et al.), while complete loss of NimC1 demonstrated cooperativity between NimC1 and Eater in the recognition and phagocytosis of bacteria (MELCARNE et al.).

Egr has also been reported to have a role in regulating phagocytosis of bacteria (Schneider 2007). In the course of studying the role of the Egr in cell competition, where apoptosis is non-cell autonomously induced, we found that dying cells appeared to persist in wing discs from egr ${ }^{3}$ mutant larvae (Fig. 1A-D). That cell death was still induced in egr ${ }^{3}$ mutant cells suggested that Egr is not required for the cells to die under these two conditions. However, because dead cells are typically cleared within 2-4 hours from wild-type wing imaginal disc epithelia (MILAN et al.), the persistence of Cas-3 positive cells that we observed in egr ${ }^{3}$ mutants suggested that loss of egr might impair corpse clearance. This prompted us to examine plasmatocytes in the lymph glands, the major larval hematopoietic organ, from WT and egr ${ }^{3}$ mutant larvae. We immunostained 
the lymph glands from both genotypes with anti-NimC1 antibodies, a mixture of P1a and $\mathrm{P} 1 \mathrm{~b}$ antibodies that specifically recognizes the phagocytic plasmatocytes of the larva (KURUCZ et al.). As a control, we also examined larvae that carried Hml-RFP, consisting of a hemocyte-specific enhancer/promoter from the Hemolectin gene fused to red fluorescent protein (RFP) that identifies larval hemocytes (CLARK et al.)(Fig. 2A). NimC1 is expressed at high levels on the plasma membrane of numerous cells in the primary lymph gland lobes from WT controls, and anti-NimC1 staining overlapped with many Hml-RFP positive cells (Fig. 2A, B). Strikingly, however, no NimC1 positive cells were evident in lymph glands from egr larvae (Fig. 2C). As the egr ${ }^{3}$ and egr ${ }^{1}$ alleles were derived from the same parental strain (IGAKI et al.), we also tested lymph glands from egr ${ }^{1}$ larvae, and again found no detectable NimC1 expression (data not shown). To examine circulating plasmatocytes, we isolated hemocytes from larval hemolymph. Although NimC1 was readily observed in circulating hemocytes from OregonR (OreR) controls (Fig. 2D), we detected no NimC1-positive hemocytes in the hemolymph from egr ${ }^{3} /$ egr $^{1}$ transheterozygous larvae, or from egr ${ }^{31}$ larvae (Fig. 2E-F). egr ${ }^{31}$ is a precise excision of the regg $1^{G S 9830} \mathrm{P}$-element present in the parental strain. Thus all of the egr alleles derived from the regg $1^{\mathrm{GS} 983}$ strain lacked circulating and lymph gland resident plasmatocytes that expressed NimC1.

Honti and colleagues reported that several Drosophila strains that were negative for NimC1 staining carried mutations in the NimC1 gene, which they postulated were scars of mobile element mobilization (HONTI et al.). Genomic sequencing of these P1-negative strains identified two independent micro-deletions in the NimC1 gene, including a 6 bp deletion between nucleotides 2264 to 2270 (HONTI et al.). Another deletion of 355 bp was found between nucleotides 1582 to 1937, accompanied by a 5 bp insertion.

Together, Honti et al found that the $355 \mathrm{bp}$ deletion and the $5 \mathrm{bp}$ insertion generated a frameshift mutation in both the NimC1 RA and $R B$ transcripts, resulting in new sequences and a premature stop codon (HONTI et al.). The alterations were predicted to give rise to a truncated NimC1 protein that lacks the intracellular and transmembrane domains and four extracellular NIM repeats, which would account for its absence on the plasma membrane of hemocytes (HONTI et al.).

To determine whether the egr ${ }^{1}$ and egr ${ }^{3}$ mutants carried mutations at the NimC1 locus, we carried out genomic sequencing of a 1254 bp region that encompasses most of the NimC1 open reading frame (Fig. 3). Our data shows that both egr ${ }^{1}$ and egr ${ }^{3}$ contain identical microdeletions and insertions within the NimC1 gene, consistent with 
their common parental origin. Each mutant strain has the same 355 bp deletion, 5 bp micro-insertion, and 6 bp micro-deletion described by Honti et al at residues 2264-2270 (Fig. 3B, C). In addition, using primers flanking the larger, 355 bp deletion in PCR reactions, we found that both the $R e g g 1^{G S 9830}$ and egr ${ }^{31}$ strains carried similar lesions. Since these egr mutants were both NimC1 negative (Fig. 2E, F), they very likely also carry the premature stop codon generated by the $355 \mathrm{bp}$ deletion and 5 bp insertion. Altogether, these results suggest that these NimC1 polymorphisms were present in the parental strain (Fig. 3D).

To restore the wild-type NimC1 locus to the egr mutants, we outcrossed both egr ${ }^{1}$ and $e g r^{3}$ to the Ore $R$ wild-type strain and isolated recombinants with the WT NimC1 locus and either the egr ${ }^{1}$ or egr ${ }^{3}$ mutation (see Methods). We then sequenced the NimC1 locus in these outcrossed egr alleles (hereafter called egr $^{1 A G}$ and egr ${ }^{3 A G}$ ) to verify that the recombination removed the mutant sequences. Both the egr ${ }^{1 A G}$ and egr ${ }^{3 A G}$ strains lacked the deletions and micro-insertions that characterized the original egr ${ }^{1}$ and egr $^{3}$ alleles (Fig. 3A). Consistent with the loss of the deletions, hemocytes from the egr ${ }^{1 A G}$ and egr ${ }^{3 A G}$ mutants regained NimC1 positivity (Fig. 2G and data not shown).

Our sequencing data thus confirm that the egr ${ }^{1}$ and egr ${ }^{3}$ mutants also have mutations at the NimC1 locus. Since the large deletion and micro-insertion in exon 3 of NimC1 also exists in both the original parental line, egr $r^{R e g g 1}$ and in egr ${ }^{31}$, a precise excision of the Regg $1^{G S 9830} \mathrm{P}$-element, it is highly likely that the NimC1 mutations in each of these egr alleles are derived from the parental strain. These NimC1 mutations are recessive (HONTI et al.), and their presence on each egr mutant chromosome in our experiments might explain the transiently persistent dying cells; perhaps they also account for the infection susceptibility found previously in egr ${ }^{3}$ mutants (SCHNEIDER et al.). Consistent with our sequencing results, the genetic backgrounds of the egr ${ }^{1}$ and egr ${ }^{3}$ alleles were previously noticed to harbor anomalies that led to egr-independent susceptibility to infection by Gram-positive bacteria (NARASIMAMURTHY et al. 2009). Complete deletion of NimC1 has been reported to prevent phagocytosis of latex beads or yeast zymosan particles by plasmatocytes (MELCARNE et al.), but whether and how phagocytosis of dying cells may be impaired by the NimC1 mutations we found here remains to be determined. NIM repeats are thought to mediate protein-protein interactions and clustering of receptors are proposed to be key in phagocytic removal of apoptotic cells (SHKLYAR et al.). If the truncated mutant NimC1 proteins are aberrantly secreted into the hemolymph, as predicted (HONTI et al.), they could interfere with critical 
NIM interactions. As the egr ${ }^{1}$ and egr ${ }^{3}$ alleles have been used in numerous studies of immunity and cell death, it may be worthwhile to re-evaluate some of the phenotypes obtained with these alleles.

\section{Methods}

Fly strains and husbandry. Flies were raised at $25^{\circ} \mathrm{C}$ on standard cornmeal-molasses food supplemented with fresh dry yeast. The following strains were used: egr ${ }^{1}$, egr $^{3}$ and egr Regg1 (IGAKI et al.), egr ${ }^{31}$ (gift of H. Kanda), egr ${ }^{3 A G}$, egr ${ }^{1 A G}$ (generated in this work), UAS-egr'R (gift of M. Miura), hml-RFP/CyO (gift of K. Brückner), yw hsflp ${ }^{122}$ (gift of G. Struhl), act $>y^{S T O P}>\mathrm{Gal} 4$ and $t u b>M y c^{S T O P}>\mathrm{Gal} 4$ (DE LA COVA et al.), OregonR (from Bloomington Drosophila Stock Center).

Cell death assays. Eggs from appropriate crosses were collected on yeasted grape plates for 2-4 hours and allowed to develop at $25^{\circ} \mathrm{C}$ in a humid chamber for 24 hours. At hatching, larvae were transferred to food vials supplemented with fresh yeast paste at densities of $<50$ larvae/vial to prevent crowding. To generate 'loser' clones in a competitive context, a $t u b>M y c^{S T O P}>$ Gal4 cassette (> represents a FLP-recognition target (FRT) site) was used to excise the $>M y c^{S T O P}$ cassette and generate tub>Gal4, UAS-GFP expressing "loser" cells in WT and in egr ${ }^{3}$ mutants. Clones were allowed to grow in wing discs for 24 hours, as described (DE LA COVA et al. ; MEYER et al. ; ALPAR et al.). FLP recombinase, under heat shock (HS) control, was activated by $\mathrm{HS}$ of larvae at $37^{\circ} \mathrm{C}$ for 10 minutes, at 48 hours after egg laying (AEL). Post-HS, larvae were allowed to grow at $25^{\circ} \mathrm{C}$ for 24 or 48 hours. To generate Myc-expressing clones, act $>y^{S T O P}>$ Gal 4 was used to generate act>Gal clones that expressed UAS-GFP and UAS-Myc in WT and in egr mutants. act>Gal4 clones were induced in larvae with a $\mathrm{HS}$ at $37^{\circ} \mathrm{C}$ for 6 min. Wing discs were dissected from larvae at 48 hours after clone induction (ACl). A detailed protocol is available upon request.

Larval dissection and imaging. Wing imaginal discs were dissected from third instar larvae as indicated above, and fixed in 4\% paraformaldehyde in phosphate-buffered saline (PF-PBS) for 20 min at room temperature and washed with $0.01 \%$ Tween-20 in PBS (PBTw). Larval carcasses were stained with Rabbit anti-Cleaved Caspase-3 (Cas3) at 1:100 (Cell Signaling). Secondary antibodies were purchased from Jackson Immunoresearch. Hoechst 33258 was used to stain DNA. Lymph glands were stained with plasmatocyte-specific P1 antibodies (anti-NimC; 1:100) (KURUCZ et al. 2007) as described below for hemocytes. Wing discs and lymph glands were mounted in VectaShield Antifade on glass slides. Images were acquired with a Zeiss Axiophot microscope with Apotome and processed using ImageJ and Adobe Photoshop.

Hemocyte immunohistochemistry and image processing. Hemocytes were collected from 10-20 experimental larvae, by bleeding from a small tear in the posterior cuticle into a 10-fold volume of PBS. Cells were then transferred to a coverslip and allowed to settle for 30 minutes at room temperature in a humidity chamber. All subsequent steps were performed directly on the coverslip. Cells were fixed in 4\% PFA in PBS for 7 minutes at room temperature, washed 3 times in PBS, permeablized for 5 minutes in $1 \%$ triton in PBS (PBT), blocked for 5 minutes in 10\% normal goat serum (NGS) in PBT and then incubated with primary antibody in $10 \%$ NGS in PBT either overnight at $4^{\circ} \mathrm{C}$ or for 30 
minutes at room temperature. Washes were carried out in PBT and secondary incubation was performed for 30 minutes at room temperature in $10 \%$ NGS in PBT. Cells were then washed 2 times in PBT, followed by 2 washes in PBS. A final 5 minute incubation with DAPI in PBS was performed. Coverslips were mounted in Slowfade Gold Antifade (Molecular Probes). Primary antibodies used were plasmatocyte-specific antiP1 (1:100) (KURUCZ et al. 2007). Secondary antibodies were Alexa Fluor antibodies (1:500, Invitrogen). Images were collected on a Zeiss Axio Imager M1 and were processed using ImageJ and Adobe Photoshop.

Genomic sequencing of egr alleles. Genomic DNA was isolated from homozygous adult female flies. $100 \mathrm{ng}$ of DNA was amplified using the primer sets as described in Honti et al. 2013. The 355bp deletion was found in the fragment amplified by P11189fw (CGCAGGAGCCTACGATAATC) and P11189rev (AAGGAATGTGGACACCATAG). The 34bp insertion was detected by the primers P1utan600fw

(AACTGGATCGTCTAACAAGT) and P1utan300rev (GGATTGATTAACCACACAGA). The fragments were cloned into a $\mathrm{PCR} \mathrm{T}^{\mathrm{TM}}$ 4-TOPO-TA vector for sequencing using the common sequencing primers M13. Primers are listed in Table 1.

Outcrossing and genotyping of egr alleles. egr ${ }^{1}$ and egr ${ }^{3}$ alleles (hereafter egr) were treated identically. egr mutant virgin females were crossed to OregonR (OreR) males and the resulting $\mathrm{F} 1$ heterozygous egr/+ virgin females were backcrossed to Ore $R$ males. Ten F2 males (either egr/+ or $+/+$ ) were singly crossed to virgin Ore $R$ females, sacrificed and used for single-fly PCR (GLOOR et al.) to identify males carrying the egr alleles, but no longer carrying the 355bp deletion in NimC1. A single egr-positive, NimC1-negative line for each allele was carried forward by crossing F3 virgin females back to Ore $R$ males. This process was repeated four times, after which egr alleles were re-isolated by crossing to +; Sco/CyO actin-GFP, a chromosome II marker/balancer strain similarly crossed into an Ore $R$ background. These alleles were named egr ${ }^{1 A G}$ and $e g r^{3 A G}$. Genotyping of $e g r^{1}$ and $e g r^{3}$ used primers flanking the reported deletion in each strain: Egr_F1 (CCAGAGCCCACTGTATCACC) and Egr_R3

(TCACCTC̄CTTTTGGAACTCG) amplify a $\sim 1500 \mathrm{bp}$ and $\sim 2000 \mathrm{bp}$ fragment in egr ${ }^{1}$ and egr ${ }^{3}$, respectively. Genotyping of NimC1 used primers flanking the 355bp deletion and 5bp insertion between nucleotides 1582 to 1937 (Honti genome annotation). Nimrod_del_F1 (CCGGGCTACGTAATGAGAAA) and Nimrod_del_R1 (CAATTTGĀGTGCGGAACCTC) amplify a 656bp fragment in $\bar{W}$ T $\overline{\text { animals and a }} \sim 300 \mathrm{bp}$ fragment in animals bearing the NimC1 deletion. Primers are listed in Table 1.

\section{Acknowledgements}

We are grateful to Chris Cary for technical assistance, and members of the Johnston lab for advice. We thank Masayuki Miura, Tatsushi Igaki, Hiroshi Kanda and Iswar Hariharan for providing fly stocks, and István Andó for the gift of anti-NimC1 antibodies. We are indebted to the Bloomington Drosophila Stock Center (BDSC) (NIH P40OD018537) and Flybase (ATTRILL et al.) for their services. Funding for this work was provided by the NIH RO1GM078464 and NCI RO1CA192838 (to LAJ) and the NSF (Graduate Research Fellowship to ARG). The egr ${ }^{3 A G}$ and egr ${ }^{1 A G}$ alleles, both WT for NimC1, were isolated by ARG in the lab of Iswar Hariharan and will be deposited at the BDSC for general use. 


\section{References}

Abrams, J. M., K. White, L. I. Fessler and H. Steller, 1993 Programmed cell death during Drosophila embryogenesis. Development 117: 29-43.

Alpar, L., C. Bergantinos and L. A. Johnston, 2018 Spatially Restricted Regulation of Spatzle/Toll Signaling during Cell Competition. Dev Cell 46: 706-719 e705.

Attrill, H., K. Falls, J. L. Goodman, G. H. Millburn, G. Antonazzo et al., 2016 FlyBase: establishing a Gene Group resource for Drosophila melanogaster. Nucleic Acids Res 44: D786-792.

Chung, Y. S., and C. Kocks, 2011 Recognition of pathogenic microbes by the Drosophila phagocytic pattern recognition receptor Eater. J Biol Chem 286: 26524-26532.

Clark, R. I., K. J. Woodcock, F. Geissmann, C. Trouillet and M. S. Dionne, 2011 Multiple TGF-beta superfamily signals modulate the adult Drosophila immune response. Curr Biol 21: 1672-1677.

de la Cova, C., M. Abril, P. Bellosta, P. Gallant and L. A. Johnston, 2004 Droso phila myc regulates organ size by inducing cell competition. Cell 117: 107-116.

Franc, N. C., P. Heitzler, R. A. Ezekowitz and K. White, 1999 Requirement for croquemort in phagocytosis of apoptotic cells in Drosophila. Science 284: 1991-1994.

Gloor, G. B., C. R. Preston, D. M. Johnson-Schlitz, N. A. Nassif, R. W. Phillis et al., 1993 Type I repressors of P element mobility. Genetics 135: 81-95.

Honti, V., G. Cinege, G. Csordas, E. Kurucz, J. Zsamboki et al., 2013 Variation of NimC1 expression in Drosophila stocks and transgenic strains. Fly (Austin) 7: 263266.

Igaki, T., H. Kanda, Y. Yamamoto-Goto, H. Kanuka, E. Kuranaga et al., 2002 Eiger, a TNF superfamily ligand that triggers the Drosophila JNK pathway. EMBO J 21: 3009-3018.

Kocks, C., J. H. Cho, N. Nehme, J. Ulvila, A. M. Pearson et al., 2005 Eater, a transmembrane protein mediating phagocytosis of bacterial pathogens in Drosophila. Cell 123: 335-346.

Kurucz, E., R. Markus, J. Zsamboki, K. Folkl-Medzihradszky, Z. Darula et al., 2007 Nimrod, a putative phagocytosis receptor with EGF repeats in Drosophila plasmatocytes. Curr Biol 17: 649-654.

Manaka, J., T. Kuraishi, A. Shiratsuchi, Y. Nakai, H. Higashida et al., 2004 Drapermediated and phosphatidylserine-independent phagocytosis of apoptotic cells by Drosophila hemocytes/macrophages. J Biol Chem 279: 48466-48476.

Melcarne, C., B. Lemaitre and E. Kurant, 2019a Phagocytosis in Drosophila: From molecules and cellular machinery to physiology. Insect Biochem Mol Biol 109: 1-12.

Melcarne, C., E. Ramond, J. Dudzic, A. J. Bretscher, E. Kurucz et al., 2019b Two Nimrod receptors, NimC1 and Eater, synergistically contribute to bacterial phagocytosis in Drosophila melanogaster. FEBS J 286: 2670-2691.

Meyer, S. N., M. Amoyel, C. Bergantinos, C. de la Cova, C. Schertel et al., 2014 An ancient defense system eliminates unfit cells from developing tissues during cell competition. Science 346: 1258236. 
Milan, M., S. Campuzano and A. Garcia-Bellido, 1997 Developmental parameters of cell death in the wing disc of Drosophila. Proc Natl Acad Sci U S A 94: 56915696.

Moreno, E., M. Yan and K. Basler, 2002 Evolution of TNF signaling mechanisms: JNKdependent apoptosis triggered by Eiger, the Drosophila homolog of the TNF superfamily. Curr Biol 12: 1263-1268.

Narasimamurthy, R., P. Geuking, K. Ingold, L. Willen, P. Schneider et al., 2009 Structure-function analysis of Eiger, the Drosophila TNF homolog. Cell Res 19: 392-394.

Schneider, D. S., J. S. Ayres, S. M. Brandt, A. Costa, M. S. Dionne et al., 2007 Drosophila eiger mutants are sensitive to extracellular pathogens. PLoS Pathog 3: e41.

Shklyar, B., F. Levy-Adam, K. Mishnaevski and E. Kurant, 2013 Caspase activity is required for engulfment of apoptotic cells. Mol Cell Biol 33: 3191-3201.

Somogyi, K., B. Sipos, Z. Penzes, E. Kurucz, J. Zsamboki et al., 2008 Evolution of genes and repeats in the Nimrod superfamily. Mol Biol Evol 25: 2337-2347.

Sonnenfeld, M. J., and J. R. Jacobs, 1995 Macrophages and glia participate in the removal of apoptotic neurons from the Drosophila embryonic nervous system. J Comp Neurol 359: 644-652.

\section{Figure Legends}

\section{Figure 1. Dying cells may be cleared less efficiently in egr ${ }^{3}$ mutants.}

A. Clones of loser cells in a wildtype wing disc, expressing GFP and cleaved caspase 3 (Cas-3); Cas-3 channel is shown in A'.

B. Loser clones, expressing GFP and Cas-3, in a egr ${ }^{3}$ mutant wing disc; Cas-3 channel is shown in B'. Clones in A and B were examined $24 \mathrm{hrs}$ after clone induction.

C. Myc-expressing clone, marked by expression of GFP, in a wildtype wing disc. Cas-3 positive cells are shown in red. C' shows Cas-3 as a single channel.

D. Myc-expressing clone, marked by expression of GFP, in an egr mutant wing disc. Cas-3 positive cells are shown in red. D' shows Cas-3 as a single channel. Clones in C and $\mathrm{D}$ were examined $48 \mathrm{hrs}$ after clone induction.

Figure 2. Lymph glands from egr ${ }^{3}$ mutant larvae and circulating hemocytes from $e g r^{1 / 3}$ and $e g r^{31}$ mutant larvae are negative for NimC1 staining.

A. Hml-RFP (red) and NimC1 (green) are expressed in many hemocytes in lymph glands from control, yw122 larvae.

B. NimC1 (red) staining in the primary lobes of lymph glands from control larvae.

C. Lymph glands from egr ${ }^{3}$ mutant larvae have no NimC1-positive hemocytes. 
D. Circulating hemocytes from the hemolymph of OreR control larvae stain positively for NimC1 (red).

E-F. Circulating hemocytes from egr ${ }^{1 / 3}$ transheterozygous larvae $(E)$ and from egr ${ }^{31}$ mutant larvae (F) lack positivity for NimC1.

G. NimC1 staining (red) in the egr ${ }^{3 A G}$ mutant lymph glands, in which the NimC1 locus was restored to WT.

\section{Fig. 3. Summary of mutations in the NimC1 locus in various egr mutants.}

A. Schematic representation of the NimC1 locus, located at on the left arm of Chr. 2 (2L in D). White blocks represent introns and 5' and 3' untranslated regions. The sequence of the locus from $y w^{122}$ flies, used as a WT strain, and from the outcrossed egr ${ }^{3 A G}$ strain, is identical to the reference ( $D$. melanogaster version r5.23). PCR genotyping suggests that the OreR WT strain is also wild type at the NimC1 locus. Numbering is as in https://flybase.org/decoratedfasta/FBgn0259896.

B. Representation of the NimC1 locus in the egr ${ }^{1}$ and egr ${ }^{3}$ strains. Two deletions, of 355 bp and 6bp, and an insertion of $5 \mathrm{bp}$, are shown. PCR genotyping in egr ${ }^{31}$ and the parental strain, egr $r^{\text {Regg1 }}$ indicates that they also carry the 355 bp deletion (D), and likely also the $5 \mathrm{bp}$ insertion and $6 \mathrm{bp}$ deletion.

C. Representation of the NimC1 locus from Honti et al, 2013. Note that the locus numbering is slightly different than in $\mathrm{B}$, presumably due to an earlier genome annotation. The mutations are identical to those found in egr ${ }^{1}$ and $e g r^{3}$ and similar to egr ${ }^{31}$ and the parental line, egr ${ }^{\text {Regg1 }}(\mathrm{B})$.

D. Results of PCR genotyping of NimC1 in egr mutants using primers flanking the 355 bp deletion and 5 bp insertion between nucleotides 1582 to 1937 (Honti et al genome annotation).

E. Representation of Chr. 2, showing the NimC1 locus on 2L, and the egr locus on the right arm of Chr. 2 (2R). 


\section{Figures}

Figure 1.
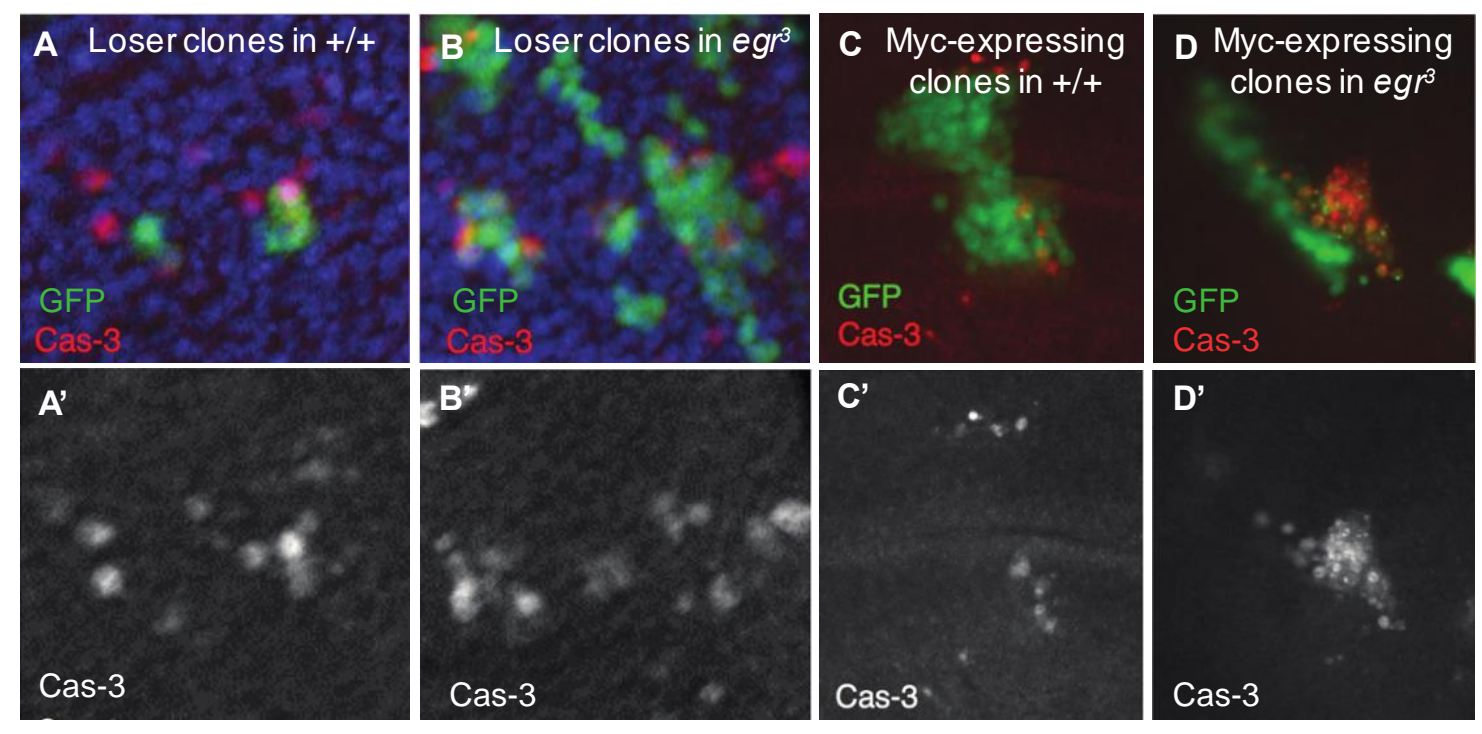

Figure 2.
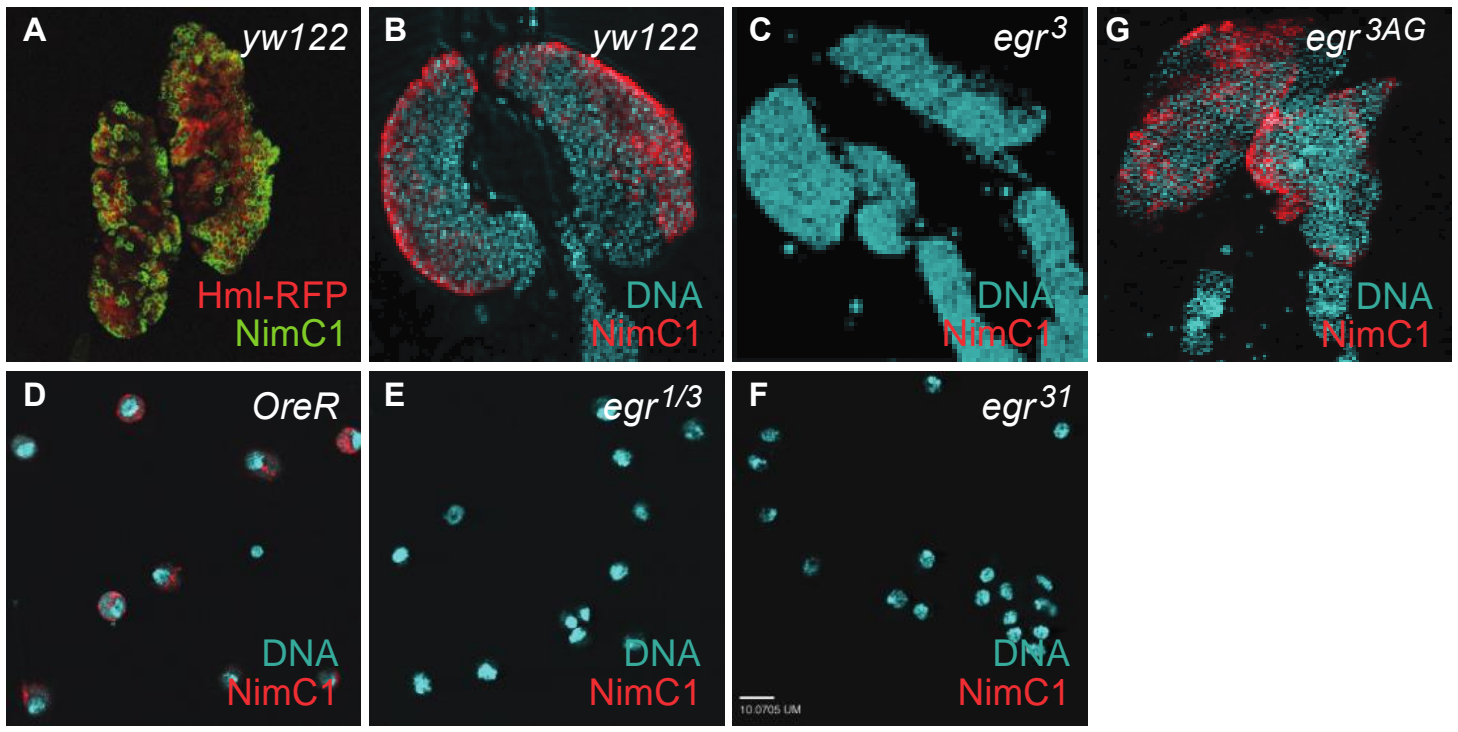
Figure 3.
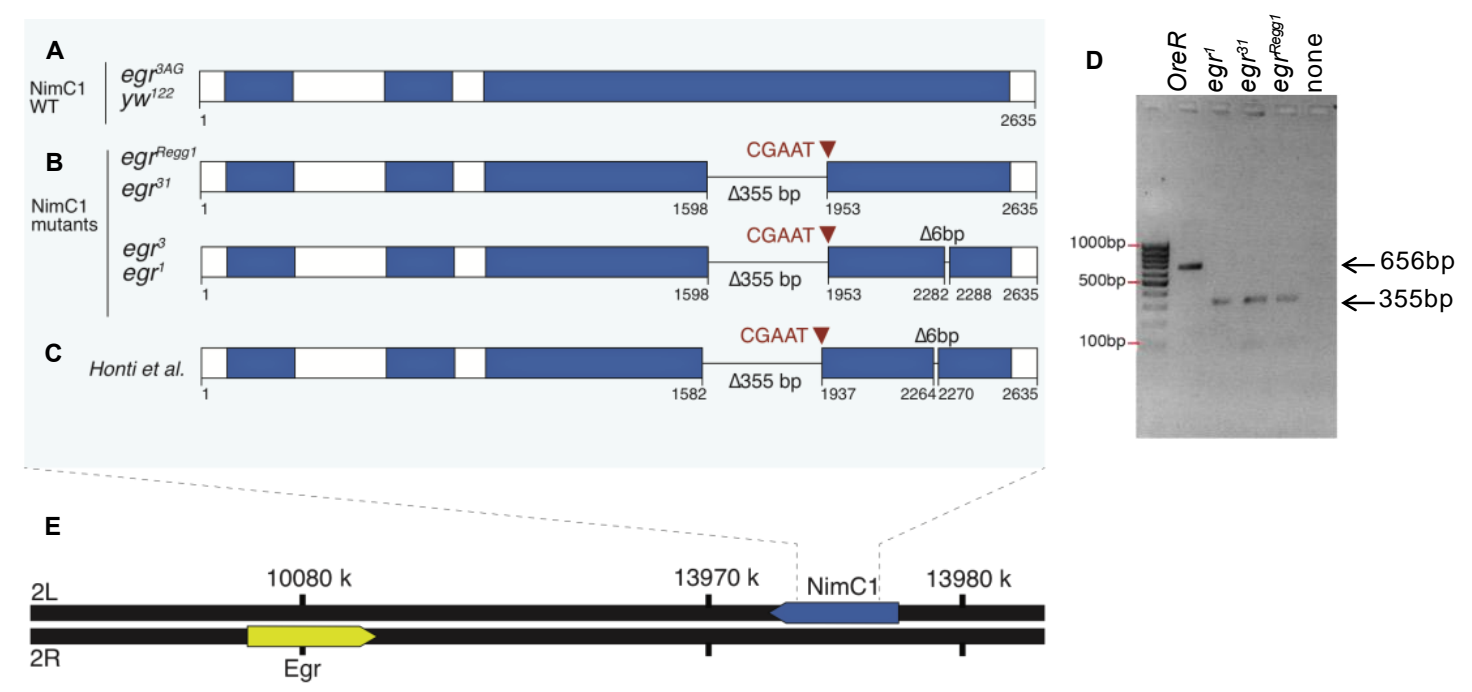

Table 1. Primers used in this study

\begin{tabular}{ll}
\hline PRIMER NAME & SEQUENCE \\
\hline P1utan600fw & AACTGGATCGTCTAACAAGT \\
P1utan300rev & GGATTGATTAACCACACAGA \\
P11189fw & AAGGAATGTGGACACCATAG \\
P11189rev & CGCAGGAGCCTACGATAATC \\
Egr_F1 & CCAGAGCCCACTGTATCACC \\
Egr_R3 & TCACCTCCTTTTGAACTCG \\
Nimrod_del_F1 & CCGGGCTACGTAATGAGAAA \\
Nimrod_del_R1 & CAATTTGAGTGCGGAACCTC
\end{tabular}

